\title{
Epidemiologia de problemas comportamentais em cães no Brasil: inquérito entre médicos veterinários de pequenos animais
}

\author{
Epidemiology of dogs behavioral problems in Brazil: a survey between small animals veterinary \\ practitioners
}

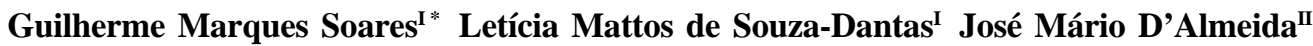 \\ Rita Leal Paixão
}

\section{RESUMO}

Problemas de comportamento afetam diretamente a qualidade de vida dos animais e das pessoas que com eles convivem. Em alguns países, os problemas de comportamento são as principais causas de abandono e eutanásia de animais de companhia. O presente estudo objetivou avaliar a prevalência dos problemas de comportamento dos cães na rotina clínica dos médicos veterinários brasileiros por meio do envio de questionários às faculdades de Medicina Veterinária do Brasil, para serem respondidos pelos profissionais responsáveis pelo atendimento clínico a cães e gatos. No total de 101 questionários respondidos, 91,1\% dos participantes afirmaram ser consultados sobre problemas de comportamento e 82,2\% afirmaram que a maior frequência das queixas comportamentais foi relacionada à espécie canina. Os respondentes, em sua maioria (56,7\%), relataram que tentaram resolver sozinhos alguns casos ou encaminharam o caso para um veterinário especializado. Dentre os respondentes, 89,1\% afirmaram não realizar eutanásia em razão de problemas de comportamento. O problema de comportamento citado como o mais frequente foi a destrutividade e, em seguida, as agressões. $O$ tratamento mais recomendado, tanto para agressões quanto para eliminações inapropriadas de urina, foi a terapia comportamental.

Palavras-chave: epidemiologia, eutanásia, agressão, bemestar do animal, comportamento animal, doenças do cão, cães.

\section{ABSTRACT}

Behavior issues directly affect animal and human welfare. In some countries, behavior problems are the main reasons for abandonment and euthanasia of companion animals. The present study aimed to investigate the prevalence of behavior problems among dogs seem by Brazilian veterinarians, by sending questionnaires to be responded by veterinarians at university referral animal hospitals in Brazil. Among 101 responses, 91.1\% of participants affirmed to be consulted about behavior issues, and $82.2 \%$ said that they mostly concern dogs. Most veterinarians (56.7\%) try to solve some behavior cases by themselves and refer others to a specialized colleague. Most affirmed to do not perform euthanasia due to behavior issues (89.1\%). The most common complaint among owners was destructive behavior, followed by aggressions. The most recommended treatment for aggressive behavior and inappropriate elimination of urine was behavior therapy.

Key words: epidemiology, euthanasia, aggression, animal welfare, animal behavior, dog diseases.

\section{INTRODUÇÃO}

A ocorrência de problemas de comportamento em cães tem implicações importantes no bem-estar animal e na relação destes com seus proprietários. Acidentes por ataques de cães são considerados um problema de saúde pública (OVERALL \& LOVE, 2001; O’SULLIVAN et al., 2008). Nos Estados Unidos da América (EUA), o custo anual do sistema público de saúde com tratamento de vítimas de ataques de cães excede cem milhões de dólares (OVERALL \& LOVE, 2001). Outro problema comum em cães é a síndrome de ansiedade de separação, que também traz sérios danos à qualidade de vida do cão e da família que o acolhe (SOARES, 2007).

IPrograma de Pós-graduação em Medicina Veterinária, Clinica e Reprodução Animal, Faculdade de Veterinária, Universidade Federal Fluminense (UFF), 24320-340, Niterói, RJ, Brasil. E-mail gsoaresvet@oi.com.br. *Autor para correspondência.

IIDepartamento de Biologia Geral, Instituto de Biologia, UFF, Niterói, RJ, Brasil.

IIIDepartamento de Departamento Fisiologia e Farmacologia, Instituto Biomédico, UFF, Niterói, RJ, Brasil. 
A agressividade canina é o problema que mais leva proprietários a procurar os serviços especializados em etologia clínica na Espanha (FATJÓ et al., 2005; FATJÓ et al., 2007). Convém destacar que, após acidentes com ataques de cães, sinais da síndrome de estresse pós-traumático foram observados em 12 de 22 crianças belgas em um estudo desenvolvido naquele país, e cinco dessas crianças desenvolveram o quadro completo da síndrome (PETERS et al., 2004; KEUSTER et al., 2006). Tal problema, por vezes, requer que a vítima e seus parentes próximos sejam encaminhados a serviços de acompanhamento psicológico (VOITH, 2009). Em estudo recente realizado em Niterói, no Estado do Rio de Janeiro, 27,6\% dos proprietários de cães de apartamento entrevistados descreveram a agressividade como um comportamento incômodo ou problemático exibido por seus cães (SOARES, 2007). No mesmo município, 87,4\% dos proprietários de cães de apartamento relataram que seus cães rosnavam ou tentavam morder em pelo menos uma situação cotidiana. A situação que desencadeava tais respostas com maior frequência ocorreu quando o cão havia sido contrariado, em 30,1\% dos casos (SOARES et al., 2007).

Cerca de vinte milhões de animais de estimação a cada ano são abandonados em abrigos nos EUA e pelo menos a metade destes é morta por causa de problemas de comportamento (SEKSEL, 1997). Sabendo-se que a maioria dos problemas de comportamento tem tratamento e que esses tratamentos, como para quaisquer doenças, exigem um diagnóstico preciso e devem ser adequados para cada problema, levando em consideração características do proprietário e de sua família. Fazem parte desse repertório terapêutico: (1) uso de medicamentos de apoio (antidepressivos e ansiolóticos, por exemplo); (2) técnicas de modificação comportamental, como a dessensibilização e o contracondicionamento; (3) técnicas de enriquecimento ambiental; e (4) modificações hormonais, que podem ser feitas por medicamentos ou por meio de cirurgias (OVERALL, 1997).

No Brasil, até o presente momento, não há uma casuística nacional dos problemas de comportamento dos cães domésticos, nem de como os casos existentes são conduzidos. O presente estudo objetivou, a partir da aplicação de questionários com médicos veterinários brasileiros, identificar os problemas de comportamento mais frequentemente atendidos por esses profissionais, assim como suas condutas clínicas e algumas consequências de tais problemas para o bem-estar e o vínculo homem-animal.

\section{MATERIAL E MÉTODOS}

Para obtenção dos dados sobre problemas de comportamento, foi utilizado o questionário de FATJÓ et al. (2006) modificado (disponível em português por meio de contato com os autores realizado via e-mail), composto de perguntas sobre cães e gatos com respostas fechadas (os resultados referentes aos felinos estão publicados em < http://jarvm.com/articles/ Vol7Iss3/Souza\%20130-137.pdf >). Após a elaboração dos questionários, estes foram enviados por correio, por meio de sistema de carta-resposta, para as 108 faculdades de Medicina Veterinária distribuídas em todo o território nacional que possuíam unidades de atendimento clínico a cães e gatos (consultórios, clínicas ou hospitais), dentre 137 instituições cadastradas no Conselho Federal de Medicina Veterinária até a data do envio dos questionários. Um novo envio foi realizado seis meses depois àquelas faculdades que não haviam respondido. Os questionários também foram enviados por correio eletrônico, para promover uma maior agilidade no envio das respostas. Todas as correspondências (inclusive o correio eletrônico) tiveram como destinatários os coordenadores de curso de Medicina Veterinária e os responsáveis pelas unidades de atendimento. As perguntas abrangeram os problemas de comportamento mais frequentes na rotina clínica de animais de companhia, a atitude dos veterinários e dos proprietários perante esses problemas e as formas de tratamento escolhidas. As perguntas e as opções de respostas estão listadas na figura 1 .

Os questionários foram respondidos por médicos veterinários, professores ou não, que prestassem atendimento nas unidades clínicas para pequenos animais, podendo um ou mais profissionais responder em cada unidade. A partir da pergunta 2, foram consideradas as respostas daqueles que afirmaram ser consultados sobre problemas de comportamento.

Os dados obtidos foram armazenados em um banco de dados do aplicativo Microsoft Office Access $2003^{\circledR}$ e analisados a partir do programa estatístico BioEstat ${ }^{\circledR}$ 5.0. Além da estatística descritiva das respostas das questões 1 , 2 e 3, foi utilizado o teste de Kruskal-Wallis, para observar se havia diferenças significativas entre as posições de classificação das respostas das questões 4 a 8 do questionário. $O$ teste de comparações Student-Newman-Keuls foi usado de forma complementar para a comparação entre os pares de respostas, para avaliar se tiveram ou não diferenças significativas.

Ciência Rural, v.40, n.4, abr, 2010. 
1- Você costuma ser consultado sobre problemas de comportamento? (marque um X ao lado da opção correta)

$\square$ Não $\square$ Sim - Que animal apresenta maior frequência? घCães $\square$ Gatos

2-Como você resolve problemas de comportamento? (marque um $\mathrm{X}$ ao lado da opção correta)

¿Etologia/Comportamento animal não é uma área da Medicina Veterinária

Eu encaminho todos os casos para um adestrador

Eu encaminho todos os casos para um veterinário especializado em comportamento animal

$\square$ Eu tento resolver alguns casos sozinho(a), e outros encaminho para um adestrador

$\square$ Eu tento resolver alguns casos sozinho(a), e outros encaminho para um veterinário especializado em comportamento animal

Eu tento resolver todos os casos sozinho(a)

3- Relacionado aos animais eutanasiados na Clínica/Hospital que você trabalha:

Em cada 10 cães eutanasiados, quantos são por problemas de comportamento? de 10

4- Quais os problemas de comportamento que são queixas mais comuns dos proprietários que você atende? (por favor, numere de 1 a 10, em ordem crescente de importância)

Agressão

Comportamento destrutivo (arranhadura/mastigação de objetos, móveis..)

Comportamentos compulsivos/ Esteriotipias (ex.: dermatite psicogênica, lambedura do pelo, etc)

Eliminações inapropriadas com fezes

Eliminações inapropriadas com urina

Falta de controle durante os passeios

Hiperatividade

Medo de pessoas ou outros animais

Medos de barulhos (fogos de artifício, trovões, barulhos fortes, visitas em casa...)

Vocalizações excessivas (latidos, choro...)

5- Em relação às queixas de agressão, por favor, enumere de 1 a 4 em ordem crescente de importância para os principais alvos para cães e gatos.

Animais da mesma espécie

Animais de outras espécies

Pessoas da Familia*

Pessoas de fora da familia*

6- Por favor, enumere de 1 a 3 (em ordem crescente de importância) que tipo de tratamento relacionado abaixo você considera mais útil para tratar distúrbios de agressividade em cães e gatos?

Castração (Ovariohisterectomia/ovariectomia/orquiectomia)

Medicamentos

_-_Terapia comportamental/modificação comportamental (ex.: mudanças ambientais, enriquecimento ambiental, etc.)

7- Por favor, enumere de 1 a 3 (em ordem crescente de importância) que tipo de tratamento relacionado abaixo você considera mais útil para tratar distúrbios de eliminação em cães e gatos?

Castração (Ovariohisterectomia/ovariectomia/orquiectomia)

Medicamentos

Terapia comportamental/modificação comportamental (ex.: mudanças ambientais, enriquecimento ambiental, etc.)

8- Quais problemas de comportamento você considera que estão mais frequentemente associados ao pedido de eutanásia ou abandono do animal por parte de proprietários? (por favor, numere de $1 \mathrm{a} 10$, em ordem crescente de importância para cães e gatos)

Agressão

Comportamento destrutivo (arranhadura/mastigação de objetos, móveis...)

Comportamentos compulsivos/ Esteriotipias (ex.: dermatite psicogênica, lambedura do pêlo, etc)

__- Eliminações inapropriadas com fezes

Eliminações inapropriadas com urina

Falta de controle durante os passeios

Hiperatividade

Medo de pessoas ou outros animais

Medos de barulhos (fogos de artifício, trovões, barulhos fortes, visitas em casa...)

Vocalizações excessivas (latidos, choro...)

Figura 1 - Perguntas e respostas do questionário modificado de FATJÓ et al. (2006) aplicado em inquérito epidemiológico realizado com médicos veterinários brasileiros, UFF, 2009.

\section{RESULTADOS E DISCUSSÃO}

Das 108 faculdades de veterinária com atendimento a pequenos animais do país, obteve-se reposta de 46 (42,6\%), totalizando 101 questionários respondidos. As regiões do Brasil foram representadas da seguinte forma: região Sul - 13 questionários (12,9\%), correspondendo a sete faculdades das 25 com atendimento a pequenos animais da região; região Sudeste - 65 questionários $(64,4 \%)$, totalizando 27 universidades entre as 58 com atendimento médicoveterinário da região; região Centro-oeste - quatro questionários $(4,0 \%)$, correspondendo a quatro instituições entre 12; região Nordeste - 16 questionários 
(15,8\%), totalizando seis entre 10 instituições; e região Norte - três questionários (3,0\%), uma universidade dentre as três faculdades existentes com unidade de atendimento. Com base na taxa de retorno dos questionários e na distribuição equilibrada das respostas por todo território nacional, a amostra foi considerada representativa em relação à experiência e à conduta dos clínicos veterinários em relação aos problemas de comportamento de cães no Brasil.

A decisão de realizar esta pesquisa com médicos veterinários de hospitais e unidades de atendimento veterinário de faculdades e universidades, ao invés de clínicas privadas, e o duplo envio das cartasrespostas podem ter contribuído para uma maior taxa de retorno, em comparação com trabalhos envolvendo questionários, como o de FATJÓ et al. (2006), os quais obtiveram taxa de resposta de $15 \%$. Pode-se presumir que os veterinários envolvidos no ambiente de ensino tenham maior predisposição a contribuir com uma pesquisa científica. Além disso, o envio adicional do questionário por correio eletrônico contornou a possível deficiência na distribuição dos questionários não só pelos correios, mas também pelos setores de comunicação de algumas instituições, o que pode ter levado ao extravio de algumas correspondências. Outra razão para a escolha das faculdades como alvo da pesquisa era obter uma distribuição nacional mais homogênea, evitando concentrar o perfil dos resultados em uma só região.

Dos 101 questionários respondidos, 91,1\% dos médicos veterinários disseram ser consultados sobre problemas de comportamento. Quanto à espécie mais consultada, $83(90,2 \%)$ médicos veterinários afirmaram ser mais consultados sobre distúrbios de comportamento em cães do que em gatos $(5,4 \%)$, e quatro respondentes (4,3\%) não identificaram a espécie com maior frequência para esse tipo de queixa. Em concordância com a literatura internacional, a maioria dos participantes afirmou ser consultada por problemas de comportamento (BEAVER, 1992; OVERALL, 1997; FATJÓ et al., 2006), ficando evidente que questões relacionadas ao comportamento animal fazem parte da rotina de médicos veterinários em todo o país.

Em relação às condutas dos médicos veterinários, 57,6\% disseram que tentam resolver alguns casos e outros são encaminhados para um veterinário especializado em comportamento animal; $21,7 \%$ disseram tentar resolver alguns casos e encaminhar outros casos para um adestrador; e 17,4\% disseram que tentam resolver sozinhos(as) qualquer caso. Apenas 3,3\% afirmaram encaminhar todos os casos para um veterinário especializado, e 1,1\% afirmaram que Etologia não é um campo da Medicina
Veterinária. Nenhum clínico disse encaminhar todos os casos para um adestrador. Em estudos que levantaram essa questão em outros países, uma maior parcela dos profissionais se mostra relutante a indicar casos para outros veterinários, ou por falta de comunicação apropriada com os colegas, ou simplesmente por temer perder a clientela (FATJÓ et al., 2006), fatores que podem estar presentes no Brasil. Todavia, não se pode descartar a possibilidade de que de muitos médicos veterinários não reconheçam ou não valorizem os problemas relacionados ao comportamento animal, negligenciando-os (CASE, 1988). Além disso, o pequeno número de profissionais que trabalham com comportamento animal, especificamente na área clínica, pode dificultar a indicação do paciente (OVERALL, 1997; FATJÓ et al., 2006) e fazer com que os clínicos tentem resolver alguns casos sozinhos. Outra hipótese para um número tão alto de possíveis indicações a especialistas seria a resposta politicamente correta, visto que o número de profissionais com esse perfil ainda é muito reduzido no país e, dessa forma, a resposta pode refletir o ideal e não o que acontece no dia-a-dia.

Quanto à morte induzida em consequência de problemas de comportamento, 82 (89,1\%) afirmaram que, em cada 10 procedimentos realizados, nenhum ocorre por queixas comportamentais, e oito $(8,7 \%)$ afirmaram que um é devido a esse tipo de queixa. Houve duas respostas $(2,2 \%)$ de que duas em cada 10 eutanásias são decorrentes de problemas comportamentais. Na questão que solicitava que o médico veterinário indicasse qual o comportamento que mais motiva o proprietário a abandonar ou solicitar a eutanásia do seu cão, 54 (58,7\%) respostas caracterizaram a agressão como muito frequente (notas 10, 9 ou 8), e 38 (41,3\%) indicaram os comportamentos destrutivos como muito frequentes. A agressão foi apontada como a causa mais frequente de abandono ou eutanásia em razão de problemas comportamentais, apresentando diferenças significativas em relação aos demais problemas listados. A destrutividade teve diferença significativa em relação a defecações inapropriadas, à falta de controle nos passeios e a medo de pessoas e barulhos.

No Brasil, aparentemente há uma cultura de não matar o cão em razão de distúrbios de comportamento, o que fica bem evidente nesta pesquisa, já que 89,1\% citaram não realizar eutanásia de animais por esse motivo, principalmente em comparação com o trabalho de FATJÓ et al. (2006), que encontraram apenas cerca de $25 \%$ de profissionais dizendo não submeter animais domésticos à eutanásia por problemas de comportamento. Diante desse 
resultado, pode-se concluir que, no Brasil, os problemas comportamentais acarretam principalmente em abandono dos animais, enquanto na Espanha, em eutanásia. Tal resultado mostra-se coerente com o que tem sido publicado (GORODETSKY, 1997; RUGBJERG et al., 2003; FATJÓ et al., 2005; FATJÓ et al., 2007; O’SULLIVAN et al., 2008), mostrando a agressão como principal motivador de abandono. Tal fato aumenta a responsabilidade dos médicos veterinários em orientar os proprietários de cães em relação à socialização e à educação de seus animais, visando ao bem-estar de todos, dos animais, dos proprietários e da sociedade (SEKSEL, 1997; SERRA, 2005).

Os médicos veterinários responderam que comportamentos destrutivos eram as queixas mais frequentes, seguidas pelas agressões. Os comportamentos com queixas menos frequentes foram: defecações em locais inapropriados; medo de pessoas e falta de controle durante os passeios (Figura 2). O teste de Kruskal-Wallis apontou diferença significativa entre as respostas $(\mathrm{P}<0,01)$ para essa questão. $\mathrm{Na}$ comparação entre os pares, agressões e comportamentos destrutivos não apresentaram diferença significativa $(\mathrm{P}=0,8)$. Ambos mostraram-se significativamente diferentes em relação: a defecações e micções inapropriadas, à falta de controle nos passeios e a medo de pessoas e de barulhos $(\mathrm{P}<0,05)$. Além destas, os comportamentos destrutivos tiveram diferença significativa em relação à vocalização excessiva $(\mathrm{P}=0,03)$. Comportamentos compulsivos tiveram diferença significativa em relação a medo de pessoas e à falta de controle nos passeios $(\mathrm{P}<0,01)$. Esta teve diferenças significativas também em relação: à hiperatividade, a medo de barulhos e a vocalizações excessivas $(\mathrm{P}<0,05)$. Hiperatividade foi significativamente diferente de medo de pessoas $(\mathrm{P}=0,01)$ e nenhum dos demais pares apresentou diferenças significativas. Os problemas mais frequentemente relatados foram idênticos aos de FATJÓ et al. (2006), já quanto aos menos frequentes só houve coincidência na falta de controle durante os passeios. As agressões aparecem em segundo lugar como queixa mais frequente, porém foi o mais incriminado como causa de abandono, o que está de acordo com o fato de que as agressões são os comportamentos que mais levam os proprietários a buscar atendimentos especializados em etologia clínica em todo o mundo (RUGBJERG et al., 2003; DENENBERG et al., 2005; BAMBERGER \& HOUPT, 2006; FATJÓ et al., 2007).

Os alvos mais frequentes das agressões caninas foram outros cães (30,4\%), seguidos de pessoas da família $(23,9 \%)$, assim como pessoas desconhecidas $(23,9 \%)$. Os resultados do teste de
Kruskal-Wallis no grupo de respostas a essa pergunta não tiveram diferença significativa $(\mathrm{P}=0,2)$. Alguns estudos apresentam os próprios familiares como principais alvos das agressões caninas (FATJÓ et al., 2005; BAMBERGER \& HOUPT, 2006). O presente estudo mostra outros cães como principais alvos dessas agressões, o que está de acordo com um estudo de RUGBJERG e et al. (2003), na Dinamarca, e com FATJÓ et al. (2006), na Espanha.

$\mathrm{O}$ tratamento mais recomendado para as agressões caninas foi a terapia comportamental, significativamente diferente da castração e do uso de medicamentos $(\mathrm{P}<0,01)$. Isso também ocorreu em relação ao tratamento para as eliminações inapropriadas com urina, em que a terapia comportamental foi a principal recomendação, apresentando diferença significativa em relação à castração $(\mathrm{P}<0,01)$ e ao uso de medicamentos $(\mathrm{P}=0,01)$. O tratamento mais recomendado para os dois problemas propostos no questionário foi a terapia comportamental, o que está de acordo com o estudo espanhol (FATJÓ et al, 2006). Deve-se considerar que a etologia clínica ainda é uma área pouco difundida no Brasil e o que questionário apresenta a questão de maneira genérica, citando mudanças ambientais e enriquecimento ambiental, mas sem categorizar que tipo de terapia seria proposta. A realização de novos estudos para identificar que tipo de terapia comportamental tem sido recomendada no país para os problemas propostos faz-se necessária, até porque a maioria dos respondentes afirmaram tentar resolver alguns casos sozinhos.

O clínico geral que atende animais de companhia é normalmente o primeiro profissional consultado sobre problemas comportamentais, o que aumenta a importância em se intensificar a formação em etologia clínica nas faculdades brasileiras de Medicina Veterinária, a fim de reduzir o número de animais mortos ou abandonados em decorrência de tais problemas, aumentar o bem-estar dos animais de companhia e melhorar o vínculo do ser humano com seu animal de estimação.

\section{CONCLUSÃO}

O médico veterinário brasileiro atende queixas de problemas comportamentais de cães em sua rotina e raramente recomenda eutanásia em virtude desses problemas. A queixa mais frequente foi relativa a comportamentos destrutivos, seguidos por comportamentos agressivos, os quais, por sua vez, foram indicados como causa mais frequente de abandono ou eutanásia dos cães. 


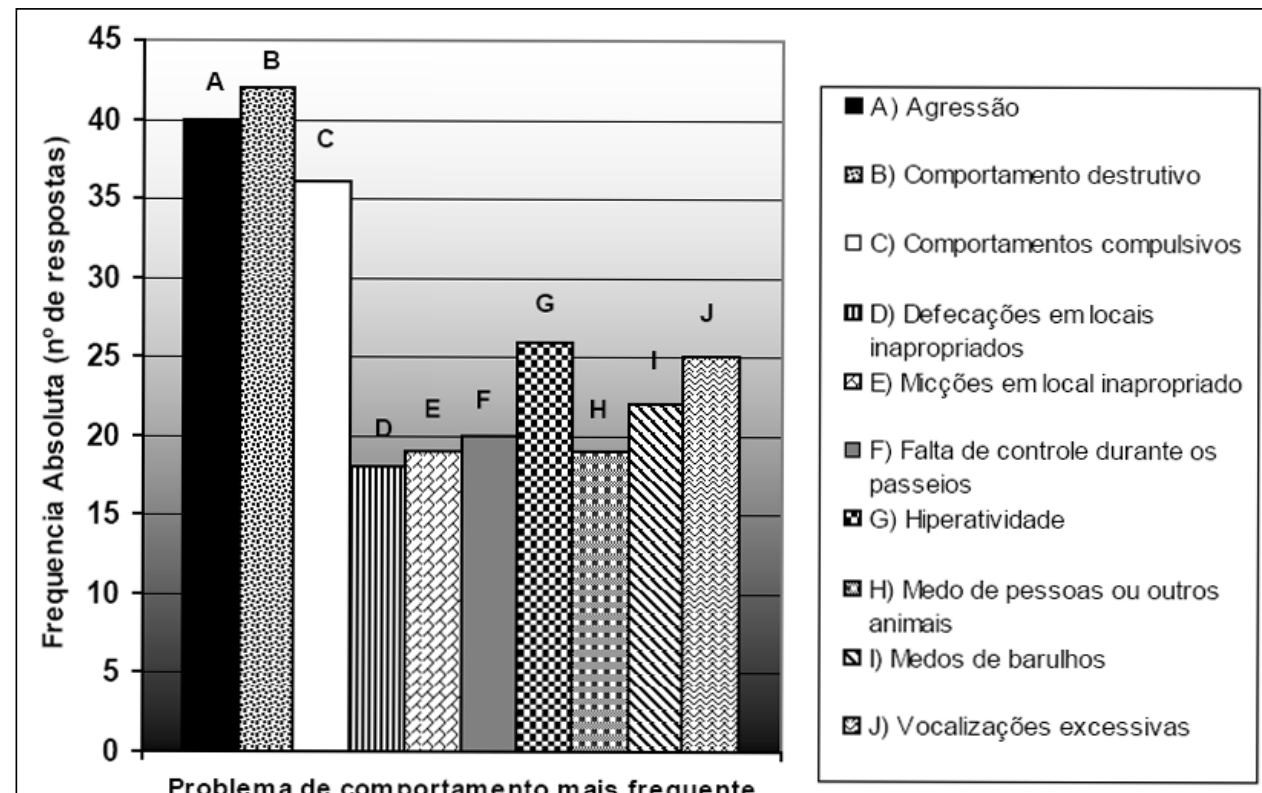

Figura 2 - Problemas de comportamento mais frequentes relatados em inquérito epidemiológico realizado com médicos veterinários brasileiros, UFF, 2009.

\section{AGRADECIMENTOS}

Agradecemos aos coordenadores de curso, diretores de unidades de atendimento e, principalmente, a todos os médicos veterinários que generosamente responderam aos questionários, tornando possível essa pesquisa.

\section{COMITÊ DE ÉTICA E BIOSSEGURANÇA}

Todos os respondentes assinaram um Termo de Consentimento Livre e Esclarecido aprovado, assim como o projeto em sua íntegra, conforme as normas do Comitê de Ética em Pesquisa (CNM/HUAP \# 096/07) da Universidade Federal Fluminense.

\section{REFERÊNCIAS}

BAMBERGER, M.; HOUPT, K.A. Signalment factors, comorbidity, and trends in behavior diagnoses in dogs: 1644 cases (1991-2001). Journal of the American Veterinary Medical Association, v.229, n.10, p.1591-1601, 2006. Disponível em: <http://dx.doi.org/10.2460/javma.229.10.1591>. Acesso em: 12 jan. 2010. doi: 10.2460/javma.229.10.1591.

BEAVER, B.V. Feline behavior: a guide for veterinarians. Philadelphia: Saunders, 1992. 276p.

CASE, D.B. Survey of expectations among clients of three small animal clinics. Journal of the American Veterinary Medical Association, v.192, p.498-502, 1988.

DENENBERG, S. et al. A comparison of cases referred to behaviorists in three different countries. In: MILLS, D. et al. Current issues and research in veterinary behavioral medicine. Indiana: Purdue University, 2005. 300p., p.56-62. (Papers presented at the INTERNATIONAL VETERINARY BEHAVIOR MEETING, 5.).
FATJÓ, J. et al. Aggression in dogs: analysis of 761 cases. In: MILLS, D. et al. Current issues and research in veterinary behavioral medicine. Indiana: Purdue University, 2005. 300p., p.251-254. (Papers presented at the INTERNATIONAL VETERINARY BEHAVIOR MEETING 5.).

FATJÓ, J. et al. The epidemiology of behavioral problems in dogs and cats: a survey of veterinary practitioners. Animal Welfare, n.15, p.179-185, 2006.

FATJÓ, J. et al. Analysis of 1040 cases of canine aggression in a referral practice in Spain. Journal of Veterinary Behavior, v.2, n.5, p.158 -165, 2007. Disponível em: <http://dx.doi.org/ 10.1016/j.jveb.2007.07.008>. Acesso em: 12 jan 2010. doi:10.1016/j.jveb.2007.07.008.

GORODETSKY, E. Epidemiology of dog and cat euthanasia across Canadian prairie provinces. Canadian Veterinary Journal, v.38, p.649-652, 1997. Disponível em: <http:// www.ncbi.nlm.nih.gov/pmc/articles/PMC1576864/pdf/ canvetj00095-0057.pdf>. Acesso em: 12 jan. 2010.

KEUSTER, T.D. et al. Epidemiology of dog bites: a Belgian experience of canine behavior and public health concerns. Veterinary Journal, n.172, p.482-487, 2006. Disponível em: <http://dx.doi.org/10.1016/j.tvjl.2005.04.024>. Acesso em: 12 jan. 2010. doi:10.1016/j.tvjl.2005.04.024.

O'SULLIVAN, E.N. et al. Characteristics of 234 dog bite incidents in Ireland during 2004 and 2005. Veterinary Record, v.163, n.2, p.37-42, 2008. Disponível em: <http:// veterinaryrecord.bvapublications.com/cgi/content/full/163/2/ 37>. Acesso em: 12 jan. 2010.

OVERALL, K.L. Clinical behavioral medicine for small animals. St. Louis: Mosby, 1997. 544p. 
OVERALL, K.; LOVE, M. Dog bites to humans - a demography, epidemiology, injury, and risk. Journal of the American Veterinary Medical Association, v.218, n.12 p.1923-1934, 2001. Disponível em: <http://dx.doi.org/10.2460/ javma.2001.218.1923>. Acesso em: 12 jan. 2010 doi: 10.2460/javma.2001.218.1923

PETERS, V. et al. A. Posttraumatic stress disorder after dog bites in children. Journal of Pediatrics, n.144, p.121-122, 2004. Disponível em: <http://dx.doi.org/ 10.1016/ j.jpeds.2003.10.024>. Acesso em: 12 jan. 2010. doi:10.1016/ j.jpeds.2003.10.024.

RUGBJERG, H. et al. Risk factors associated with interdog aggression and shooting phobias among purebred dogs in Denmark. Preventive Veterinary Medicine, n.58, p.85100, 2003. Disponível em: <http://dx.doi.org/10.1016/S01675877(03)00011-4>. Acesso em: 12 jan. 2010. doi:10.1016/ S0167-5877(03)00011-4.

SEKSEL, K. Puppy socialization classes. Veterinary Clinics of North America: Small Animal Practice, v.27, n.3, p.465475, 1997.
SERRA, C.M. Avaliação de um método para socialização primária de filhotes de cães domésticos e seus efeitos nos participantes. 2005. 87f. Dissertação (Mestrado em Medicina Veterinária) - Instituto de Veterinária, Universidade Federal Rural do Rio de Janeiro, Seropédica, RJ.

SOARES, G.M. Levantamento da presença de sinais de ansiedade de separação em cães de apartamento em Niterói-RJ. 2007. 80f. Dissertação (Mestrado em Medicina Veterinária) - Programa de Pós-graduação em Medicina Veterinária (Clínica e Reprodução Animal), Universidade Federal Fluminense, Rio de Janeiro, RJ.

SOARES, G.M. et al. Agressividade em cães de apartamento no município de Niterói-RJ. Revista da Universidade Rural Série Ciências da Vida, v.27, p.323-325, 2007.

VOITH, V.L. The impact of companion animal problems on society and the role of veterinarians. Veterinary Clinics of North America: Small Animal Practice, v.39, n.2, p.327-345, 2009. Disponível em: <http://dx.doi.org/10.1016/j.cvsm.2008.10.014>. Acesso em: 12 jan. 2010. doi:10.1016/j.cvsm.2008.10.014. 